\title{
Serum TFF3 may be a pharamcodynamic marker of responses to chemotherapy in gastrointestinal cancers
}

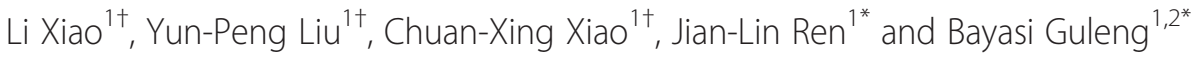

\begin{abstract}
Background: As a secreted protein, serum trefoil factor 3 (TFF3) has been reported to be a biomarker of several malignancies. We further investigated whether TFF3 can be applied as a biomarker for and predictor of responses to chemotherapy in gastrointestinal cancer.
\end{abstract}

Methods: Serum and urine samples were collected from 90 patients with gastric cancer, 128 patients with colorectal cancer and 91 healthy individuals. Serum and urine TFF3 levels were measured using an ELISA.

Results: Serum and urine TFF3 levels were significantly higher in the patients with gastric and colorectal cancer compared with the healthy individuals $(P<0.05)$. Higher serum levels of TFF3 were significantly correlated with distant metastasis and an advanced stage in the two types of cancer $(P<0.05)$. Age and the number of lymph node metastases were significantly correlated with serum TFF3 levels in colorectal cancer, and decreased serum TFF3 levels were significantly correlated with responses to chemotherapy in both the gastric and the colorectal cancer partial response (PR) groups. A combination of serum and urine data did not significantly improve the detection of either cancer, although urine levels have shown a significant negative relationship with the glomerular filtration rate (GFR).

Conclusions: Our data indicate that TFF3 may be an effective biomarker of tumor stage and the presence of distant metastasis, and may be a pharmacodynamic marker of response to chemotherapy in gastrointestinal cancer.

Keywords: Gastric cancer, Colorectal cancer, TFF3, Biomarker

\section{Background}

Gastric cancer is the fourth most common cancer worldwide and has the third highest mortality rate, accounting for $11 \%$ of cancer-related deaths [1]. Colorectal cancer is the third most common cancer globally, accounting for $7.6 \%$ of cancer-related deaths worldwide [2].

Cancer outcome is highly dependent on the stage at which the disease is detected. Unfortunately, clinical symptoms mostly arise at a late stage, when the disease has already spread outside of the gastric and colorectal region [3]. Surgical excision remains the main treatment, but overall 5-year survival is limited. However, although chemotherapy plays an important role for those patients

\footnotetext{
* Correspondence: renjianl@xmu.edu.cn; bayasi8@gmail.com

${ }^{\dagger}$ Equal contributors

'Department of Gastroenterology, Zhongshan Hospital affiliated to Xiamen University, 201 Hubin South Road, Xiamen 361004, Fujian Province, China ${ }^{2}$ Faculty of Clinical Medicine, Medical College of Xiamen University, Xiangan South Road, Xiangan District, Xiamen 361102, Fujian Province, China
}

with advanced gastric and colorectal cancer, conventional chemotherapeutic agents have yielded a treatment bottleneck [4]. Therefore, novel biomarkers for better cancer detection, diagnosis and therapeutic prediction are urgently needed.

Trefoil factor family (TFF) members are small secreted proteins that are co-expressed with mucins by the epithelial cells lining the gastrointestinal tract [5]. In humans, three members of the TFF have been identified, and their functions are thought to center on their role in mucosal protection, namely, their interactions with mucins and the stimulation of cell motility [6-8]. TFF3 or intestinal trefoil factor (ITF) is expressed in the goblet cells of the intestine and shows limited expression outside of the gastrointestinal tract, in the breast, salivary gland, hypothalamus and respiratory tract [6].

TFF3 is overexpressed in a variety of human malignancies, including gastric and colorectal cancer, and has

\section{Biomed Central}


demonstrated prosurvival, proinvasive and proangiogenic activities $[9,10]$. Secreted proteins can be used as biomarkers to identify and characterize diagnosis, prognosis and potential therapeutic responses. Body fluids such as plasma, serum and urine, tissue specimens and cancer cell lines have been utilized extensively toward this goal [11].

As a secreted protein, serum TFF3 has been reported to be a biomarker for several malignancies [12-16]. In this study, we investigated whether serum and urine TFF3 levels could be applied as biomarkers for gastrointestinal cancer and predictors of responses to chemotherapy. Our data demonstrated that serum TFF3 can be applied as an effective biomarker for the detection of tumor stages and distant metastasis and pharamcodynamic marker of responses to chemotherapy in gastrointestinal cancer. Urine TFF3 is a different indicator than serum levels and could also be a biomarker for the early detection of renal dysfunction.

\section{Methods}

\section{Ethics statement}

This study was approved by the Ethics Committee (No: 20081009) of Zhongshan Hospital, affiliated with Xiamen University in Xiamen, Fujian Province, China. Written consent was obtained from all participants who were involved in this study.

\section{Patients and healthy control characteristics}

In total, 90 patients with gastric cancer, 128 patients with colorectal cancer and 91 relatively age- and gendermatched healthy individuals who underwent health examinations from September 2011 to April 2013 at Zhongshan Hospital were enrolled in this study. Both biopsies and surgical specimens were histologically confirmed to be gastric and colorectal adenocarcinomas. The patients were classified by age, gender, site, the degree of differentiation, lymph node metastasis, distant metastasis and clinical stages. Clinical and pathological information was obtained from a patient database at Zhongshan Hospital, affiliated with Xiamen University. We obtained blood samples from the 218 patients before treatment. Among these patients, 46 with gastric cancer and 57 with colorectal cancer contributed urine samples at the same time. Tables 1 and 2 show the backgrounds of the patients and the clinical characteristics of the gastric and colorectal cancer groups, respectively. In total, 91 healthy individuals (male/female of $51 / 40$ and age of $50.1 \pm 9.9$ y) were used as controls. The patients in the cancer group were older, and there were a higher number of male patients in the gastric cancer group. Notably, for most populations, these sex differences in gastric cancer are in both high- and lowincidence regions [17]. In this study, 24 patients with advanced gastric cancer and 29 patients with advanced colorectal cancer received chemotherapy. From the chemotherapy group, we obtained blood and urine samples before
Table 1 Background of gastric cancer patients with serum TFF3 analysis

\begin{tabular}{|c|c|c|c|}
\hline Parameters & Cases (n) & Serum TFF3 (ng/ml) & $P$ \\
\hline \multicolumn{4}{|l|}{ Age } \\
\hline$\leq 60$ & 44 & $12.72 \pm 1.21$ & \multirow[t]{2}{*}{0.0529} \\
\hline$>60$ & 46 & $20.29 \pm 3.59$ & \\
\hline \multicolumn{4}{|l|}{ Gender } \\
\hline Male & 75 & $16.65 \pm 2.28$ & \multirow[t]{2}{*}{0.9489} \\
\hline Female & 15 & $16.31 \pm 2.90$ & \\
\hline \multicolumn{4}{|l|}{ Site } \\
\hline Fundus/Cardia & 35 & $13.96 \pm 1.18$ & \\
\hline Gastric body & 22 & $20.28 \pm 7.43$ & \multirow[t]{2}{*}{0.323} \\
\hline Antrum & 30 & $16.92 \pm 1.69$ & \\
\hline \multicolumn{4}{|c|}{ Degree of differentiation } \\
\hline Well-moderately & 41 & $13.10 \pm 1.27$ & \multirow[t]{2}{*}{0.1034} \\
\hline Poorly & 49 & $19.51 \pm 3.40$ & \\
\hline Stage & & $(|-I|$ VS IV) & \multirow[t]{5}{*}{0.0017} \\
\hline 1 & 9 & $8.89 \pm 1.35$ & \\
\hline$\|$ & 9 & $12.94 \pm 2.24$ & \\
\hline III & 29 & $12.07 \pm 0.73$ & \\
\hline IV & 43 & $22.02 \pm 3.89$ & \\
\hline \multicolumn{4}{|c|}{ Lymphatic metastasis } \\
\hline$N(-)$ & 13 & $10.71 \pm 1.01$ & \multirow[t]{3}{*}{0.3057} \\
\hline$N(+)$ & 33 & $12.23 \pm 0.83$ & \\
\hline N1 & 12 & $12.91 \pm 1.81$ & \\
\hline $\mathrm{N} 2$ & 7 & $11.84 \pm 1.98$ & \multirow[t]{2}{*}{0.9603} \\
\hline N3 & 4 & $11.85 \pm 0.82$ & \\
\hline \multicolumn{4}{|l|}{ Distant metastasis } \\
\hline MO & 48 & $11.97 \pm 0.76$ & \multirow[t]{2}{*}{0.0108} \\
\hline M1 & 42 & $21.88 \pm 3.97$ & \\
\hline
\end{tabular}

and after chemotherapy (two cycles later). We then applied the RECIST 1.1 criteria for solid tumors to evaluate the efficacy of chemotherapy.

\section{RECIST 1.1 criteria for the assessment of the efficacy of chemotherapy}

Target lesions were evaluated as follows. Complete response (CR): The disappearance of all target lesions. Any pathological lymph nodes (whether target or non-target) must have a reduction in the short axis of $<10 \mathrm{~mm}$. Partial response (PR): At least a 30\% decrease in the sum of the diameters of the target lesions, using the baseline sum of the diameters as a reference. Progressive disease (PD): At least a $20 \%$ increase in the sum of the diameters of the target lesions, using the smallest sum in the study as a reference (including the baseline sum if that is the smallest in the study). In addition to the relative increase of $20 \%$, the sum must also demonstrate an 
Table 2 Background of colorectal cancer patients with serum TFF3 analysis

\begin{tabular}{|c|c|c|c|}
\hline Item & Cases (n) & Serum TFF3 (ng/ml) & $P$-value \\
\hline \multicolumn{4}{|l|}{ Age } \\
\hline$\leq 60$ & 70 & $13.09 \pm 0.95$ & 0.0061 \\
\hline$>60$ & 58 & $19.20 \pm 2.11$ & \\
\hline \multicolumn{4}{|l|}{ Gender } \\
\hline Male & 75 & $16.14 \pm 1.42$ & 0.7699 \\
\hline Female & 53 & $15.47 \pm 1.82$ & \\
\hline \multicolumn{4}{|l|}{ Site } \\
\hline Colon & 67 & $15.86 \pm 1.57$ & 0.9957 \\
\hline Rectum & 61 & $15.47 \pm 1.82$ & \\
\hline \multicolumn{4}{|c|}{ Degree of differentiation } \\
\hline Well-moderately & 107 & $20.66 \pm 3.74$ & 0.057 \\
\hline Poorly & 21 & $14.92 \pm 1.11$ & \\
\hline Stage & & $(I-I \mid I V S$ IV) & 0.0007 \\
\hline । & 11 & $11.32 \pm 2.21$ & \\
\hline$\|$ & 30 & $12.20 \pm 0.88$ & \\
\hline III & 25 & $14.27 \pm 2.89$ & \\
\hline IV & 45 & $21.82 \pm 2.39$ & \\
\hline \multicolumn{4}{|c|}{ Lymphatic metastasis } \\
\hline$N(-)$ & 46 & $11.86 \pm 0.79$ & 0.1786 \\
\hline$N(+)$ & 33 & $14.70 \pm 2.22$ & \\
\hline N1 & 19 & $10.45 \pm 1.09$ & 0.00229 \\
\hline N2 & 14 & $20.47 \pm 1.98$ & \\
\hline \multicolumn{4}{|l|}{ Distant metastasis } \\
\hline MO & 84 & $12.86 \pm 0.99$ & 0.001 \\
\hline M1 & 44 & $21.59 \pm 2.44$ & \\
\hline
\end{tabular}

absolute increase of at least $5 \mathrm{~mm}$. Note that the appearance of one or more new lesions is also considered to be progression. Stable disease (SD): Neither sufficient shrinkage to qualify for PR nor a sufficient increase to qualify for PD, using the smallest sum of the diameters in the study as a reference [18].

\section{ELISA analysis}

Serum TFF3 levels were measured by a human TFF3 ELISA kit (R\&D Systems, Minneapolis, MN). It is measured using method of double antibody sandwich and antibodies concentration as follow: $360 \mathrm{ug} / \mathrm{ml}$ of mouse anti-human TFF3 as a capture antibody and $18 \mathrm{ug} / \mathrm{ml}$ of biotinylated sheep anti-human TFF3 as a detection antibody. Capture antibody has been pre-coated onto a microplate. Prior to the ELISA, $1 \mathrm{ml}$ of blood was collected from each healthy individual and each patient, followed by centrifugation for serum separation. All samples and standards were assayed in duplicate. Briefly, the capture antibody was diluted to the working concentration in
PBS. A 96-well microplate was coated with $100 \mu \mathrm{l}$ per well of the diluted capture antibody $(2.0 \mu \mathrm{g} / \mathrm{ml})$. The plate was sealed and incubated overnight at room temperature. The plates were blocked by adding $300 \mu \mathrm{l}$ of reagent diluents (1\%BSA in PBS, PH7.2-7.4) and incubating at room temperature for 1 hour. Next, $100 \mu \mathrm{l}$ of sample $(1 \mathrm{~g} / \mathrm{l})$ or standards in reagent diluents were added to each well and incubated for 2 hours at room temperature. The undiluted standard serves as the high standard $(100 \mathrm{ng} / \mathrm{ml})$, reagent diluents serves as the zero standard, produce a 2 -fold dilution series $(1.56 \mathrm{ng} / \mathrm{ml}$, $3.12 \mathrm{ng} / \mathrm{ml}, 6.25 \mathrm{ng} / \mathrm{ml}, 12.5 \mathrm{ng} / \mathrm{ml}, 25 \mathrm{ng} / \mathrm{ml}, 50 \mathrm{ng} / \mathrm{ml}$ ) between them. After repeated aspiration and washing, $100 \mu \mathrm{l}$ of the detection antibody $(100 \mathrm{ng} / \mathrm{ml})$ was added and incubated for 2 hours at room temperature. After repeated aspiration and washing, $100 \mu \mathrm{l}$ of the working dilution of streptavidin-HRP was added to each well and incubated for 20 minutes at room temperature. Next, $100 \mu \mathrm{l}$ of substrate solution (1:1 mixure of $\mathrm{H}_{2} \mathrm{O}_{2}$ and Tetramethylbenzidine) was added for a 20-minute incubation, followed by the addition of $50 \mu \mathrm{l}$ of stop solution $\left(2 \mathrm{mmol} / \mathrm{l}, \mathrm{H}_{2} \mathrm{SO} 4\right)$. The absorbance at $450 \mathrm{~nm}$ was measured. Concentrations of human TFF3 in the serum samples were calculated from the standard curves of recombinant human TFF3.

Urine TFF3 levels were measured by a human TFF3 ELISA kit (Cusabio, IL). This assay employs the quantitative sandwich enzyme immunoassay. Antibody specific for TFF3 has been pre-coated onto a microplate. For this assay, $1 \mathrm{ml}$ of urine was collected from healthy individuals and from patients. Add $100 \mu \mathrm{l}$ of standards $(0 \mathrm{ng} / \mathrm{ml}$, $1.56 \mathrm{ng} / \mathrm{ml}, 3.12 \mathrm{ng} / \mathrm{ml}, 6.25 \mathrm{ng} / \mathrm{ml}, 12.5 \mathrm{ng} / \mathrm{ml}, 25 \mathrm{ng} / \mathrm{ml}$, $50 \mathrm{ng} / \mathrm{ml}, 100 \mathrm{ng} / \mathrm{ml})$ and $100 \mu \mathrm{l}$ of sample $(0.08 \mathrm{~g} / \mathrm{l})$ per well. After incubation of 2 hours at $37^{\circ} \mathrm{C}$, remove the liquid of each well and add $100 \mu \mathrm{l}$ of biotin antibody $(1 \times)$ to each well, incubate 1 hour at $37^{\circ} \mathrm{C}$, then aspirate and wash 3 times with washing buffer. Add $100 \mu$ of HRPavidin $(1 \times)$ to each well and incubate 1 hour at $37^{\circ} \mathrm{C}$, then wash 5 times and add $90 \mu \mathrm{l}$ of TMB Substrate to each well. Incubate $15-30$ minutes at $37^{\circ} \mathrm{C}$ and avoid from light. Then add $50 \mu \mathrm{l}$ of stop solution and the absorbance at $450 \mathrm{~nm}$ was measured. Concentrations of human TFF3 in the urine samples were calculated from the standard curves of recombinant human TFF3.

\section{Assessment of the glomerular filtration rate (GFR)}

In this study, 99 Tc-DTPA clearance was defined as the serum activity of 99 Tc-DTPA at 1 and 3 hours following the injection of 99 Tc-DTPA, and the results were calculated using a constant body surface area of $1.73 \mathrm{~m}^{2}(\mathrm{ml} / \mathrm{min} /$ $1.73 \mathrm{~m}^{2}$ ).

\section{Statistical analysis}

Statistical analyses were performed using SPSS v13.0 (SPSS, Chicago, IL), and graphs were generated using 


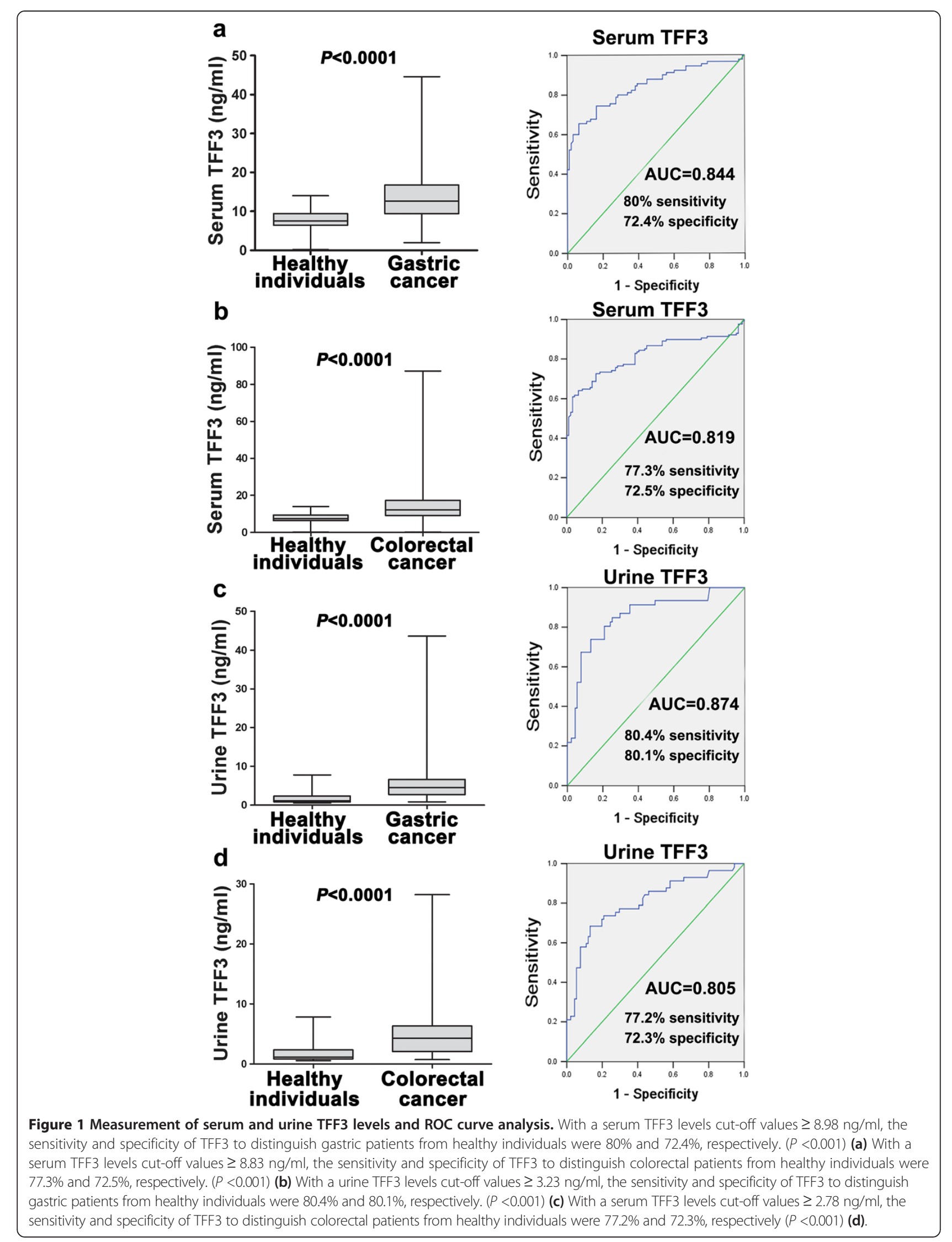


GraphPad Prism 5.0 (GraphPad Software Inc., CA). Student's $t$ test and the Kruskal-Wallis test were used to compare data between groups. All values are expressed as the mean and standard deviation (SD), and $P<0.05$ was considered to be statistically significant. Receiver operating characteristic (ROC) curves were generated to assess the diagnostic accuracy of each parameter, and the sensitivity and specificity of the optimum cutoff point were defined as those values that maximized the area under the ROC curve (AUC).

\section{Results}

Serum and urine levels of TFF3 were elevated in gastric and colorectal cancer patients compared with healthy individuals

TFF3 is a secreted protein present in the serum and urine and can be detected by ELISA. Therefore, we measured the TFF3 protein levels in serum and urine samples from gastric and colorectal cancer patients prior to treatment, including surgery, chemotherapy and radiotherapy, and from healthy individuals.

The serum TFF3 levels in the patients with gastric cancer were $16.59 \pm 1.958 \mathrm{ng} / \mathrm{ml}$, and were significantly elevated compared with $7.80 \pm 0.233 \mathrm{ng} / \mathrm{ml}$ in the group of healthy individuals (Figure 1a, $P<0.05$ ). ROC curve analysis showed that serum TFF3 had $80.0 \%$ of positive predictive value, $80 \%$ sensitivity and $72.4 \%$ specificity, with an AUC of 0.844 and optimal cut-off $(8.98 \mathrm{ng} / \mathrm{ml})$ when gastric cancer patients were separated from healthy individuals. The urine TFF3 levels in the patients with gastric cancer were significantly elevated to $6.46 \pm$ $1.046 \mathrm{ng} / \mathrm{ml}$, compared with $1.83 \pm 0.162 \mathrm{ng} / \mathrm{ml}$ in the group of healthy individuals (Figure 1c, $P<0.05$ ). ROC curve analysis showed that urine TFF3 had $73.91 \%$ of positive predictive value, $80.4 \%$ sensitivity and $80.1 \%$ specificity, with an AUC of 0.874 and optimal cut-off $(3.12 \mathrm{ng} / \mathrm{ml})$ when gastric cancer patients were separated from healthy individuals.

The serum TFF3 levels in the patients with colorectal cancer were $15.86 \pm 1.118 \mathrm{ng} / \mathrm{ml}$, and were significantly elevated compared with $7.80 \pm 0.233 \mathrm{ng} / \mathrm{ml}$ in the group of healthy individuals (Figure 1b, $P<0.05$ ). ROC curve analysis showed that serum TFF3 had $76.6 \%$ of positive predictive value, $77.3 \%$ sensitivity and $72.5 \%$ specificity, with an AUC of 0.819 and optimal cut-off $(8.83 \mathrm{ng} / \mathrm{ml})$, when colorectal cancer patients were separated from healthy individuals. The urine TFF3 levels in the patients with colorectal cancer were significantly elevated to $5.56 \pm$ $0.696 \mathrm{ng} / \mathrm{ml}$, compared with $1.83 \pm 0.162 \mathrm{ng} / \mathrm{ml}$ in the group of healthy individuals (Figure $1 \mathrm{~d}, P<0.05$ ). ROC curve analysis showed that urine TFF3 had $75.4 \%$ of positive predictive value, $77.2 \%$ sensitivity and $72.3 \%$
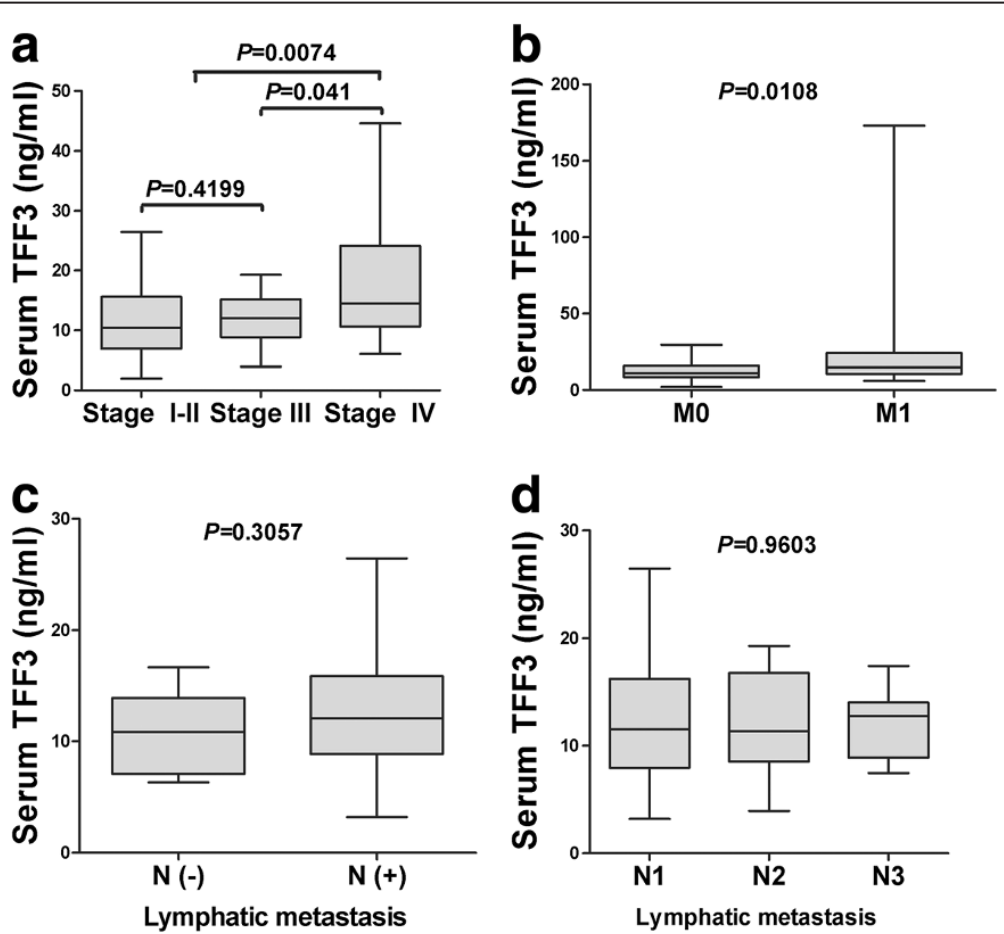

Figure 2 Measurement of serum TFF3 levels in gastric cancer patients. Serum TFF3 levels were compared in gastric cancer patients. The significant ratios with $\mathrm{P}<0.05$ between 2 groups(clinical stage III/IV) and another 2 groups (clinical stage I-II/IV) (a) The significant ratios with $\mathrm{P}<0.05$ between 2 groups M0 (patients without distant metastasis) and M1 (patients with distant metastasis) (b), lymphatic metastasis after excluding the interference of distant metastasis (c) and number of lymphatic metastases (d). 
specificity, with an AUC of 0.805 and optimal cut-off $(3.01 \mathrm{ng} / \mathrm{ml})$ when colorectal cancer patients were separated from healthy individuals. However, the combination of serum and urine data could not significantly improve the detection of both cancers compared with serum or urine TFF3 alone (AUC $<0.5$ ), and urine TFF3 did not show a significant correlation with serum levels (data not shown).

Taken together, these data indicated that serum and urine TFF3 levels can be applied as a biomarker for separate gastric and colon cancer detection.

\section{Serum TFF3 levels is correlated with the development and progression of gastric cancer}

To define the prognostic value of serum TFF3 levels in gastric cancer, we analyzed the clinicopathological parameters of gastric cancer. Our data indicated that a higher serum TFF3 level was significantly correlated with the clinical stage (I-III $v s$ IV) and distant metastasis (Figure 2a-b, $P<0.05)$. No significant difference was observed in lymphatic metastasis or the number of lymphatic metastases after excluding the interference of distant metastasis (Figure 2c-d). The serum TFF3 level was did not significantly correlated with patient gender or age, the site of the tumor (fundus/cardia, body or antrum) or the degree of differentiation (well, moderately or poorly differentiated) (Table 1 ).

\section{Serum TFF3 levels is correlated with the development and progression of colorectal cancer}

To determine the prognostic value of serum TFF3 levels in colorectal cancer, we analyzed the clinicopathological
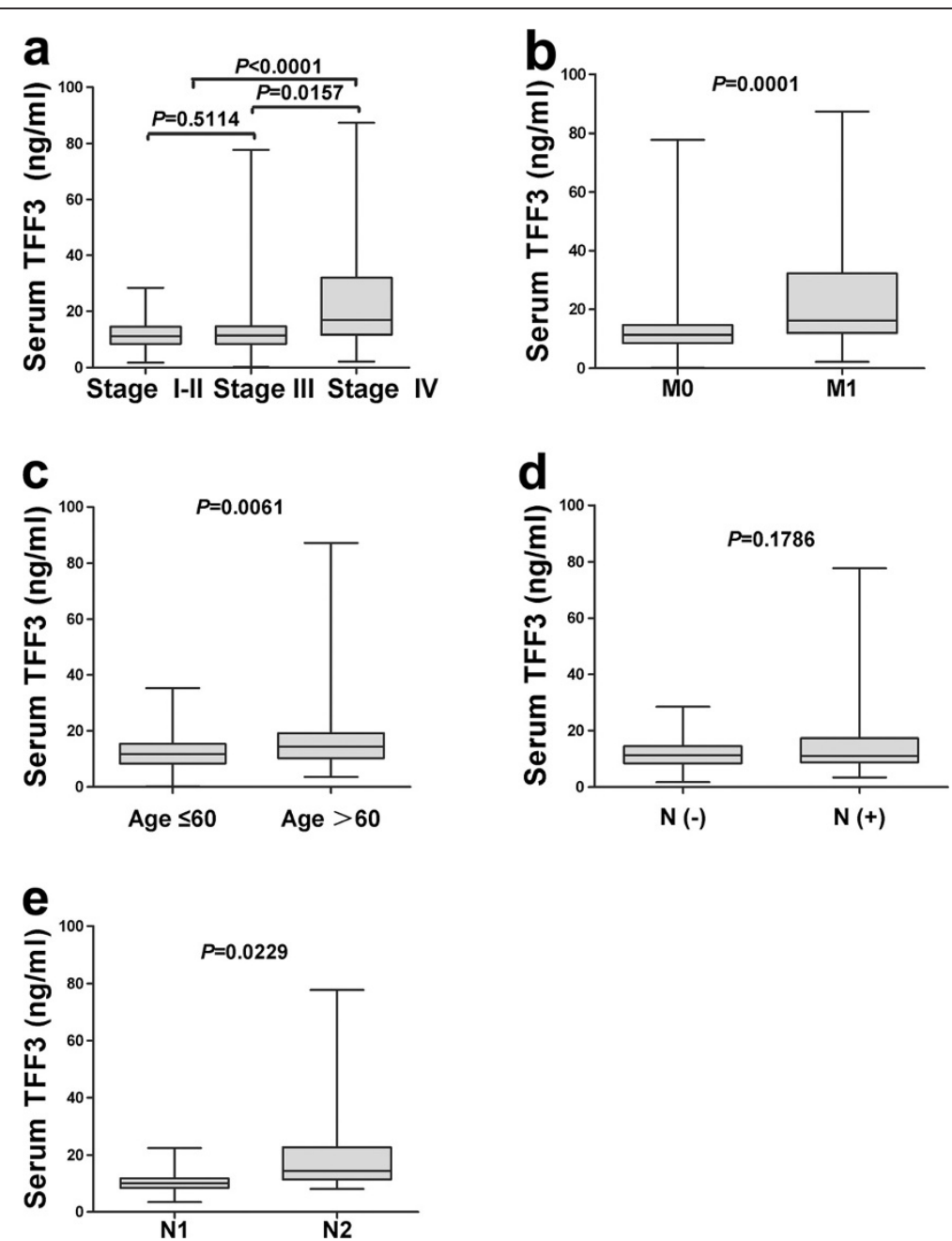

Figure 3 Measurement of serum TFF3 levels in colorectal cancer patients. Serum TFF3 levels were compared in colorectal cancer patients. The significant ratios with $\mathrm{P}<0.05$ between 2 groups (clinical stage III/IV) and another 2 groups (clinical stage I-II/IV) (a) The significant ratios with $\mathrm{P}<0.05$ between 2 groups M0 (patients without distant metastasis) and M1 (patients with distant metastasis) (b), lymphatic metastasis after excluding the interference of distant metastasis (d), The significant ratios with $\mathrm{P}<0.05$ between 2 groups (age $>60 \mathrm{y}$ and age $\leq 60 \mathrm{y}$ ) (c) and another 2 groups (N1 and N2) number of lymphatic metastases (e). 
parameters of gastric cancer. Our data demonstrated that a higher serum TFF3 level was significantly correlated with the clinical stage (I-III $v s$ IV) and distant metastasis (Figure $3 a-b, P<0.05$ ). As shown in Figure $3 c$, in contrast to gastric cancer, the serum TFF3 levels of older patients (age $>60 \mathrm{y}$ ) with colorectal cancer were higher than the levels of younger patients (age $\leq 60 \mathrm{y}, P<0.05$ ). No significant difference was observed in lymphatic metastasis after excluding the interference of distant metastasis (Figure 3d). However, different numbers of lymphatic metastases (N1 and N2) showed statistically significant differences (Figure 3e, $P<0.05$ ). The serum TFF3 level was not significantly correlated with a patient's tumor site or degree of differentiation (Table 2).

\section{Serum TFF3 levels as a pharamcodynamic marker of responses to chemotherapy in both gastric and colorectal cancer PR patients}

To define whether serum and urine TFF3 levels could be applied as predictors of responses to chemotherapy, we analyzed the relationship between serum and urine TFF3 levels before and two cycles after chemotherapy in patients with advanced gastric and colorectal cancer.
The chemotherapeutic strategy consisted of fluoropyrimidines (5-fluorouracil, [5-FU]-leucovorin and capecitabine) with oxaliplatin. In total, 24 patients with advanced gastric cancer and 29 with colorectal cancer underwent chemotherapy and were assessed using the RECIST 1.1 criteria. The advanced gastric cancer group showed an efficacy of 0/24 for CR, 6/24 for PR, 9/24 for SD and 9/ 24 for PD. Significantly higher serum TFF3 levels were observed in the PD group compared with the PR and SD groups. Interestingly, decreased serum TFF3 levels were significantly correlated with responses to chemotherapy in the gastric cancer PR group (Figure 4a, $P<0.05$ ). The advanced colorectal cancer group showed an efficacy of $0 / 29$ for CR, 8/24 for PR, 17/29 for SD and 4/ 29 for PD. Decreased serum TFF3 levels were significantly correlated with responses to chemotherapy in the colorectal cancer PR group. However, no significant differences in serum TFF3 levels were observed between the PR, SD and PD groups (Figure $4 \mathrm{~b}, P<0.05$ ). Taken together, the data indicated that the serum level of TFF3 can be applied as a pharamcodynamic marker of responses to chemotherapy in both gastric and colorectal cancer PR patients.
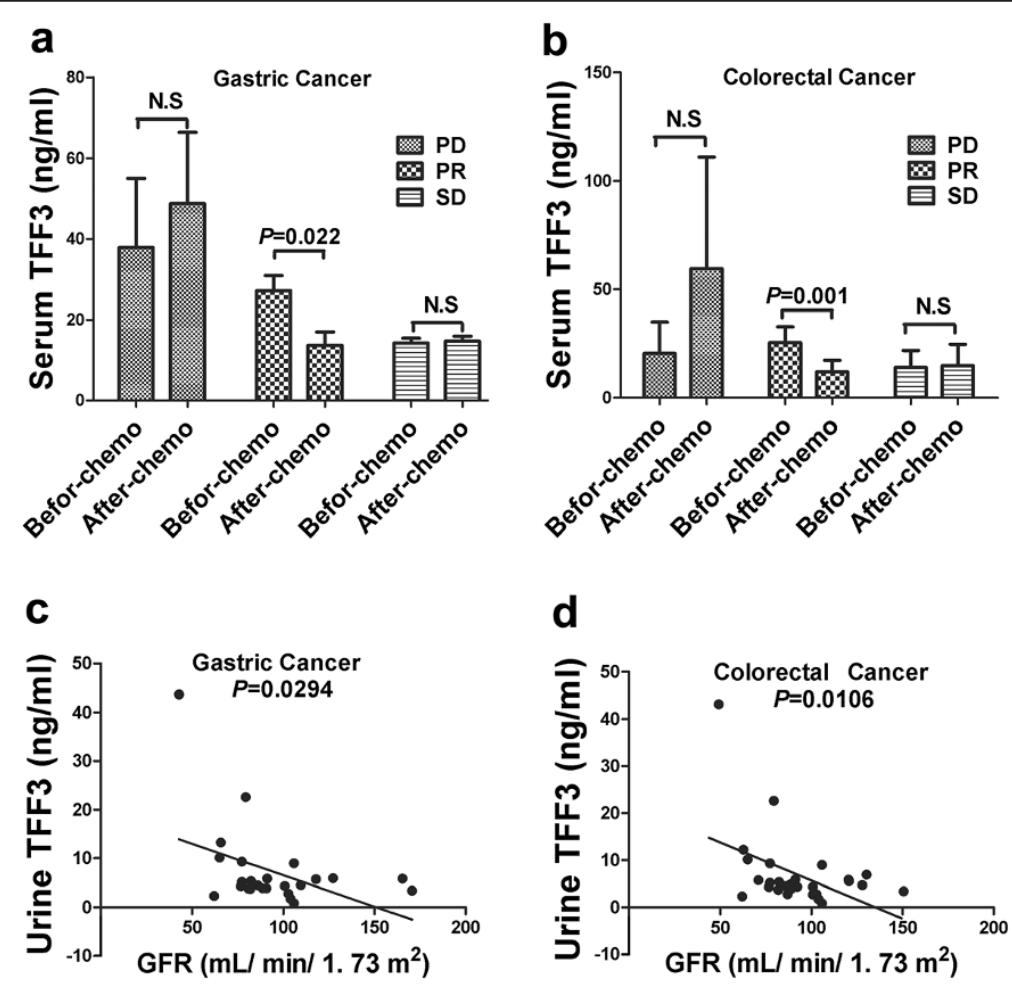

Figure 4 Serum TFF3 analysis in the chemotherapy group and correlation analysis between urine TFF3 and the GFR. (a) and (b) present an analysis of serum TFF3 levels in the PR, SD and PD groups of patients with gastric and colorectal cancer before and after chemotherapy, respectively. The significant ratios with $\mathrm{P}<0.05$ between 2 groups (before and after chemotherapy in the PR group of gastric and colorectal cancer). (c) and (d) present a correlation analysis between urine TFF3 levels and the GFR in patients with gastric and colorectal cancer, respectively. Urine TFF3 levels were significantly negatively correlated with the GFR in both the gastric and the colorectal cancer groups $(P<0.05)$. 
Urine TFF 3 levels showed a significant negative relationship with the GFR

We analyzed the relationship of urine TFF3 levels with clinicopathological parameters and responses to chemotherapy and did not find a significant difference between urine TFF3 levels based on clinical stage, lymphatic metastasis, the patient's gender or age, the site and degree of differentiation of the tumor or responses to chemotherapy for gastric or colorectal cancer (Tables 3 and 4). Therefore, we investigated whether urine TFF3 levels can reflect renal function. As shown in Figure $4 c-d$, the

Table 3 Clinicopathological parameters and responses to chemotherapy with urine TFF 3 in gastric cancer patients

\begin{tabular}{|c|c|c|c|}
\hline Parameters & Cases (n) & Urine TFF3 (ng/ml) & $P$ \\
\hline \multicolumn{4}{|l|}{ Age } \\
\hline$\leq 60$ & 24 & $4.76 \pm 0.72$ & 0.089 \\
\hline$>60$ & 22 & $8.33 \pm 1.99$ & \\
\hline \multicolumn{4}{|l|}{ Gender } \\
\hline Male & 36 & $6.82 \pm 1.30$ & 0.529 \\
\hline Female & 10 & $5.20 \pm 1.21$ & \\
\hline \multicolumn{4}{|l|}{ Site } \\
\hline Fundus/Cardia & 18 & $6.26 \pm 1.23$ & \\
\hline Gastric body & 16 & $8.47 \pm 2.54$ & 0.087 \\
\hline Antrum & 12 & $4.10 \pm 0.99$ & \\
\hline \multicolumn{4}{|c|}{ Degree of differentiation } \\
\hline Well-moderately & 27 & $6.35 \pm 0.93$ & 0.898 \\
\hline Poorly & 19 & $6.63 \pm 2.20$ & \\
\hline Stage & & $(|-|||$ VS IV) & 0.414 \\
\hline$|-| \mid$ & 9 & $4.15 \pm 0.59$ & \\
\hline III & 5 & $6.40 \pm 4.10$ & \\
\hline IV & 32 & $7.12 \pm 1.36$ & \\
\hline \multicolumn{4}{|c|}{ Lymphatic metastasis } \\
\hline$N(-)$ & 6 & $4.23 \pm 0.79$ & 0.743 \\
\hline$N(+)$ & 9 & $7.12 \pm 1.36$ & \\
\hline \multicolumn{4}{|l|}{ Distant metastasis } \\
\hline MO & 14 & $4.96 \pm 1.44$ & 0.346 \\
\hline M1 & 32 & $7.12 \pm 1.36$ & \\
\hline \multicolumn{4}{|c|}{ Responses to chemotherapy } \\
\hline$P R$ & 6 & & \\
\hline Befor-chemo & & $8.53 \pm 1.93$ & 0.189 \\
\hline After-chemo & & $5.63 \pm 0.72$ & \\
\hline SD & 9 & & \\
\hline Befor-chemo & & $5.93 \pm 0.94$ & 0.76 \\
\hline After-chemo & & $6.44 \pm 1.35$ & \\
\hline PD & 9 & & \\
\hline Befor-chemo & & $6.02 \pm 1.68$ & 0.053 \\
\hline After-chemo & & $23.05 \pm 7.44$ & \\
\hline
\end{tabular}

Table 4 Clinicopathological parameters and responses to chemotherapy with urine TFF3 in colorectal cancer patients

\begin{tabular}{|c|c|c|c|}
\hline Item & Cases (n) & Urine TFF3 (ng/ml) & $P$-value \\
\hline \multicolumn{4}{|l|}{ Age } \\
\hline$\leq 60$ & 37 & $5.71 \pm 0.94$ & 0.088 \\
\hline$>60$ & 20 & $3.99 \pm 0.89$ & \\
\hline \multicolumn{4}{|l|}{ Gender } \\
\hline Male & 37 & $5.17 \pm 0.93$ & 0.558 \\
\hline Female & 20 & $6.04 \pm 1.03$ & \\
\hline \multicolumn{4}{|l|}{ Site } \\
\hline Colon & 38 & $4.84 \pm 0.64$ & 0.201 \\
\hline Rectum & 19 & $6.75 \pm 1.66$ & \\
\hline \multicolumn{4}{|c|}{ Degree of differentiation } \\
\hline Well-moderately & 47 & $5.67 \pm 0.84$ & 0.558 \\
\hline Poorly & 10 & $4.58 \pm 0.56$ & \\
\hline Stage & & (I-III VS IV) & 0.222 \\
\hline$|-| \mid$ & 9 & $3.72 \pm 1.16$ & \\
\hline III & 11 & $4.60 \pm 1.58$ & \\
\hline IV & 37 & $6.16 \pm 0.92$ & \\
\hline \multicolumn{4}{|l|}{ Lymphatic metastasis } \\
\hline$N(-)$ & 9 & $2.92 \pm 1.00$ & 0.164 \\
\hline$N(+)$ & 14 & $5.57 \pm 1.32$ & \\
\hline \multicolumn{4}{|l|}{ Distant metastasis } \\
\hline Mo & 20 & $4.20 \pm 0.99$ & 0.184 \\
\hline \multicolumn{4}{|c|}{ Responses to chemotherapy } \\
\hline PR & 8 & & \\
\hline Befor-chemo & & $9.18 \pm 3.06$ & 0.076 \\
\hline After-chemo & & $3.06 \pm 0.93$ & \\
\hline SD & 17 & & \\
\hline Befor-chemo & & $5.95 \pm 1.16$ & 0.76 \\
\hline After-chemo & & $8.83 \pm 3.35$ & \\
\hline PD & 4 & & \\
\hline Befor-chemo & & $3.26 \pm 0.87$ & 0.13 \\
\hline After-chemo & & $9.87 \pm 3.68$ & \\
\hline
\end{tabular}

GFR of 26 gastric and 29 colorectal cancer patients was assessed, and urine TFF3 levels were significantly negatively correlated with the GFR in both the gastric and the colorectal cancer groups $(P<0.05)$.

\section{Discussion}

Gastric and colorectal cancer are the two most common malignancies worldwide [19]. Despite developed strategies of chemotherapy and radiotherapy, the curative treatment for gastric and colorectal cancer is the surgical resection of primary tumors at early stages [20,21]. Certain patients with gastric and colorectal cancer, even with the same TNM stage, have different prognoses and 
treatment responses. Therefore, new biological markers for early detection and predictors of prognosis for gastric and colorectal cancer are urgently needed in clinical work.

Secreted proteins play an important role in cell signaling, communication and migration [22,23]. The secreted protein TFF3 has malignant characteristics to promote the invasion of tumor cells by acting both directly on malignant cells and indirectly on the vasculature [24]. TFF3 is upregulated in most human malignancies, and the protein's expression is correlated with a highly aggressive phenotype and poor prognosis $[15,25,26]$. However, TFF3 has been reported by several investigators to have conflicting roles in these regards $[8,13,27,28]$.

Several earlier clinical studies identified serum TFF3 as a new marker for gastric cancer [13,29]. In previous study, a serum TFF3 level greater than $7 \mathrm{ng} / \mathrm{ml}$ indicated higher sensitivity in predicting the presence of gastric cancer $[30,31]$. In this study, we found that serum and urine TFF3 levels of the patients with gastric and colorectal cancer were significantly higher that in healthy individuals. High serum levels of TFF3 were significantly correlated with distant metastasis and an advanced stage in both types of cancer. This suggest that high TFF3 expression was significantly correlated with vein invasion, and advanced stage. TFF3 may play an important role in promoting gastrointestinal cancer development, progression and dissemination. Our data have shown that decreased serum TFF3 levels were significantly correlated with responses to chemotherapy in both the gastric and the colorectal cancer PR groups. However, no significant changes were observed in patients with PD or SD after chemotherapy. Thus, our results suggested that serum TFF3 may be a potential useful marker for patients with gastric and colorectal cancer and their metastases. It may be a pharmacodynamics marker of responses to chemotherapy in both gastric and colorectal cancer PR patients.

To investigate whether urine TFF3 can also be used as a biomarker for gastric and colorectal cancer detection, we analyzed urine and serum TFF3 levels at the same time. Interestingly, urine TFF3 levels in the patients with gastric and colorectal cancer were significantly elevated compared with the levels of the healthy individuals. However, the combination of serum and urine data could not significantly improve the detection of both cancers compared with serum or urine TFF3 data alone, and urine TFF3 levels did not show a significant correlation with serum levels. Our data also did not show a significant difference for the urine TFF3 level based on clinical stage, lymphatic metastasis, the patient's gender or age, the site or degree of tumor differentiation or responses to chemotherapy for gastric or colorectal cancer. However, urine TFF3 levels were significantly negatively correlated with the GFR in both the gastric and the colorectal cancer groups. This result suggests that the origin of urine TFF3 in gastric and colorectal cancer is much more complicated than the serum levels. TFF3 is a small peptide hormone produced by epithelial cells in multiple tissues, including the kidney. The decreased GFR in acute and chronic kidney diseases is caused by pathological damage to the kidney structure [32]. Urine TFF3 is a sensitive and specific biomarker for tubular injury used to detect early renal dysfunction [33,34]. Decreases in urinary TFF3 are associated with proximal tubular injury and correlate well with the severity of renal histopathological lesions $[35,36]$. Our results suggested that urine TFF3 is a different indicator to serum levels and that multiple factors can affect urine TFF3 levels, such as the secretion of cancer, tubular secretion and the GFR.

In summary, our data indicated that serum TFF3 can be applied as an effective biomarker for the detection of tumor stages and distant metastasis and as a predictor of responses to chemotherapy in both gastric and colorectal cancer. Urine TFF3 is a different indicator to serum levels and could also be a biomarker for the early detection of renal dysfunction.

\section{Conclusions}

In summary, our data indicated that serum TFF3 can be applied as an effective biomarker for the detection of tumor stages and distant metastasis and as a pharamcodynamic marker of responses to chemotherapy in gastrointestinal cancer. Urine TFF3 is a different indicator to serum levels and could also be a biomarker for the early detection of renal dysfunction.

\section{Abbreviations}

TFF: Trefoil factor family; ITF: Intestinal trefoil factor; SD: Stable disease; PD: Progressive disease; PR: Partial response; CR: Complete response; GFR: Glomerular filtration rate.

\section{Competing interests}

The authors declare that they have no competing interests.

\section{Authors' contributions}

$J L R$ and BG designed the experiments; LX, YPL and CXX performed the research; and $L X$ and BG wrote the paper. All the authors revised the manuscript and approved the final version.

\section{Acknowledgments}

This study was supported by National Natural Science Foundation of China (No. 81370505, 81370591, 81225025 \& 91229201) and Ministry of Health Foundation for State Key Clinical Department.

Received: 13 June 2013 Accepted: 10 June 2014 Published: 14 June 2014

\section{References}

1. Parkin DM, Bray F, Ferlay J, Pisani P: Global cancer statistics, 2002. CA Cancer J Clin 2005, 55:74-108.

2. Shin HR: Global activity of cancer registries and cancer control and cancer incidence statistics in Korea. J Prev Med Public Health 2008, 41:84-91.

3. de Wit M, Fijneman RJA, Verheul HMW, Meijer GA, Jimenez CR: Proteomics in colorectal cancer translational research: biomarker discovery for clinical applications. Clin Biochem 2013, 46:466-479. 
4. Chan MWY, Chan WY, Leung WK, Chan KK, To KF, Sung JJY: Anti-sense trefoil factor family-3 (intestinal trefoil factor) inhibits cell growth and induces chemosensitivity to adriamycin in human gastric cancer cells. Life Sci 2005, 76:2581-2592.

5. Wright NA, Poulsom R, Stamp G, Van Noorden S, Sarraf C, Elia G: Trefoil peptide gene expression in gastrointestinal epithelial cells in inflammatory bowel disease. Gastroenterology 1993, 104:12-20.

6. May FE, Westley BR: Trefoil proteins: their role in normal and malignant cells. J Pathol 1997, 183:4-7.

7. Peitz U, Wiede A, Guenther T, Ebert M, Hoffmann W, Malfertheiner P: TFF3 in esophageal, gastric mucosa as well as in gastric juice. Gastroenterology 2000, 118:A1291

8. Im S, Yoo C, Jung JH, Choi HJ, Yoo J, Kang CS: Reduced expression of TFF1 and increased expression of TFF3 in gastric cancer: correlation with clinicopathological parameters and prognosis. Int J Med Sci 2013, 10:133-140.

9. Kannan N, Kang J, Kong X, Tang J, Perry JK, Mohankumar KM: Trefoil factor 3 is oncogenic and mediates anti-estrogen resistance in human mammary carcinoma. Neoplasia 2010, 12:1041-1053.

10. Perry JK, Kannan N, Grandison PM, Mitchell MD, Lobie PE: Are trefoil factors oncogenic? Trends Endocrinol Metabol 2008, 19:74-81.

11. Makridakis M, Vlahou A: Secretome proteomics for discovery of cancer biomarkers. J Proteome 2010, 73:2291-2305

12. Lacroix M: Significance, detection and markers of disseminated breast cancer cells. Endocr Relat Cancer 2006, 13:1033-1067.

13. Aikou S, Ohmoto Y, Gunji T, Matsuhashi N, Ohtsu H, Miura H: Tests for serum levels of trefoil factor family proteins can improve gastric cancer screening. Gastroenterology 2011, 141:837-845.

14. Walker G, MacLeod K, Williams AR, Cameron DA, Smyth JF, Langdon SP: Estrogen-regulated gene expression predicts response to endocrine therapy in patients with ovarian cancer. Gynecol Oncol 2007, 106:461-468.

15. Qu Y, Yang Y, Ma D, Xiao W: Increased trefoil factor 3 levels in the serum of patients with three major histological subtypes of lung cancer. Oncol Rep 2012, 27:1277-1283.

16. Bignotti E, Ravaggi A, Tassi RA, Calza S, Rossi E, Falchetti M: Trefoil factor 3 : a novel serum marker identified by gene expression profiling in high-grade endometrial carcinomas. Br J Cancer 2008, 99:768-773.

17. Camargo MC, Goto Y, Zabaleta J, Morgan DR, Correa P, Rabkin CS: Sex hormones, hormonal interventions, and gastric cancer risk: a metaanalysis. Cancer Epidemiol Biomarkers Prev 2012, 21:20-38.

18. Eisenhauer EA, Therasse P, Bogaerts J, Schwartz LH, Sargent D, Ford R: New response evaluation criteria in solid tumours: revised RECIST guideline (version 1.1). Eur J Cancer 2009, 45:228-247.

19. Bellenir K: Cancer sourcebook : basic consumer health information about major forms and stages of cancer, featuring facts about head and neck cancers, lung cancers, gastrointestinal cancers, genitourinary cancers, lymphomas, blood cell cancers, endocrine cancers, skin cancers, bone cancers, metastatic cancers, and more; along with facts about cancer treatments, cancer risks and prevention. 6th edition. Detroit, Ml: Omnigraphics; 2011.

20. Macdonald JS, Smalley SR, Benedetti J, Hundahl SA, Estes NC, Stemmermann GN: Chemoradiotherapy after surgery compared with surgery alone for adenocarcinoma of the stomach or gastroesophageal junction. N Engl J Med 2001, 345:725-730.

21. Rustgi AK: The genetics of hereditary colon cancer. Genes Dev 2007, 21:2525-2538.

22. May M: From cells, secrets of the secretome leak out. Nat Med 2009, 15:828.

23. Kulasingam V, Diamandis EP: Strategies for discovering novel cancer biomarkers through utilization of emerging technologies. Nat Clin Pract Oncol 2008, 5:588-599.

24. May FE: The potential of trefoil proteins as biomarkers in human cancer. Biomark Med 2012, 6:301-304.

25. Meng JR, Tang HZ, Zhou KZ, Shen WH, Guo HY: TFF3 and survivin expressions associate with a lower survival rate in gastric cancer. Clin Exp Med 2013, 13:297-303

26. Vestergaard EM, Borre M, Poulsen SS, Nexo E, Torring N: Plasma levels of trefoil factors are increased in patients with advanced prostate cancer. Clin Cancer Res 2006, 12:807-812.

27. Ahmed AR, Griffiths AB, Tilby MT, Westley BR, May FE: TFF3 is a normal breast epithelial protein and is associated with differentiated phenotype in early breast cancer but predisposes to invasion and metastasis in advanced disease. Am J Pathol 2012, 180:904-916.

28. Yamachika T, Werther JL, Bodian C, Babyatsky M, Tatematsu M, Yamamura Y: Intestinal trefoil factor: a marker of poor prognosis in gastric carcinoma. Clin Cancer Res 2002, 8:1092-1099.

29. Kaise M, Miwa J, Tashiro J, Ohmoto Y, Morimoto S, Kato M: The combination of serum trefoil factor 3 and pepsinogen testing is a valid non-endoscopic biomarker for predicting the presence of gastric cancer: a new marker for gastric cancer risk. J Gastroenterol 2011, 46:736-745.

30. Yamada A, Hoteya S, lizuka T, Kaise M: Endoscopic resection of early gastric cancer. Nihon Rinsho 2012, 70:1778-1782

31. Samson $\mathrm{MH}, \mathrm{Nexo}$ E: Validation of commercial assays for measurements of trefoil factor family peptides in serum. Clin Chem Lab Med 2011, 49:2057-2060

32. Levey AS, Stevens LA: Estimating GFR using the CKD Epidemiology Collaboration (CKD-EPI) creatinine equation: more accurate GFR estimates, lower CKD prevalence estimates, and better risk predictions. Am J Kidney Dis 2010, 55:622-627.

33. Yu Y, Jin H, Holder D, Ozer JS, Villarreal S, Shughrue P: Urinary biomarkers trefoil factor 3 and albumin enable early detection of kidney tubular injury. Nat Biotechnol 2010, 28:470-477.

34. Marrer E, Dieterle F: Impact of biomarker development on drug safety assessment. Toxicol Appl Pharmacol 2010, 243:167-179.

35. Dieterle F, Sistare F, Goodsaid F, Papaluca M, Ozer JS, Webb CP: Renal biomarker qualification submission: a dialog between the FDA-EMEA and Predictive Safety Testing Consortium. Nat Biotechnol 2010, 28:455-462.

36. Blomme EA: Assessing renal function: some significant improvements on the horizon. Vet J 2011, 188:128-129.

doi:10.1186/1472-6890-14-26

Cite this article as: Xiao et al:: Serum TFF3 may be a pharamcodynamic marker of responses to chemotherapy in gastrointestinal cancers. BMC Clinical Pathology 2014 14:26.

\section{Submit your next manuscript to BioMed Central and take full advantage of:}

- Convenient online submission

- Thorough peer review

- No space constraints or color figure charges

- Immediate publication on acceptance

- Inclusion in PubMed, CAS, Scopus and Google Scholar

- Research which is freely available for redistribution 